Biol. Cybernetics 21, 79-83 (1976)

(C) by Springer-Verlag 1976

\title{
Some System Considerations of Neuron Pools with Feedback*
}

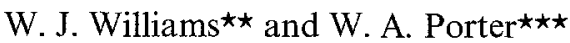 \\ Received: May 2, 1975
}

\begin{abstract}
This paper describes an approach to the analysis of the inputoutput relationships present in a neuron pool that receives a number of inputs. These inputs consist of primary inputs to the neuron pool and inputs resulting from feedback of information from the neuron pool as well. Multiple input-output relationships are obtained in terms of the synaptic weightings of the inputs, the membrane response characteristics of the neurons and the conduction delays on the feedback pathways. $y=(I+M H D)^{-1} \cdot M F x$ is the explicit representation of the cell pool behavior assuming quasi-linear conditions, where $y$ is the output vector of cell responses, $I$ is the identity matrix, $M$ is the response matrix of the cells, $H$ is the feedback synaptic weighting matrix, $D$ is the delay matrix, $F$ is the input weighting matrix, and $x$ is the input vector. It is shown that a solution to this formulation exists, is unique, is stable, and can be computed by specified algorithms. An insight gained from this formulation suggests that the output of each cell in the pool is related to virtually all of the inputs to the pool and the outputs of all cells in the pool.
\end{abstract}

\section{Introduction}

During the 1960's the coincident development of the electronic digital computer as a laboratory tool and sophisticated microelectrode recording techniques resulted in intensive study of the response properties of single neurons in a wide variety of systems: More recently interest has been shown in the mathematical details of dynamic response properties of populations of neural elements (Williams, 1968, 1969, 1972; Coggshall and Bekey, 1970; Williams and Heetderks, 1973; and Coggshall, 1973).

For many years the work of neurophysiologists has reflected an interest in cataloging the properties of neurons as means of placing them into distinctive functional categories. Recent experimental neurophysiology has also been addressed to the population response of groups of neurons, but on a single neuron analysis basis (Henneman, Somjen and Carpenter, 1965 a, 1965 b; Carpenter and Henneman, 1966; Towe, 08470 .

* Supported by NSF Grant GK 38301 and US-PHS Grant NS

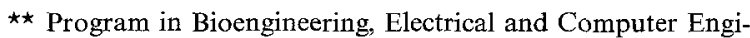
neering Department, The University of Michigan.

$\star \star \star$ Computer Information and Control Program, and Department of Electrical and Computer Engineering, The University of Michigan.
1968; Mendell and Henneman, 1971; Whitehorn et al., 1972; and Johnson, 1974).

Many studies have characterized neural behavior using linear transfer functions. This is appropriate under small signal or quasi-linear conditions. It seems clear that one thrust in theoretical neurophysiology should be in the direction of a detailed treatment of the input-output characteristics of populations of sensory motor neurons with the aim of deriving appropriate transfer functions to describe their behavior, as expressed in Cohen (1973).

In this paper we depart from previous work where the transmission of information through successively cascaded relay nuclei has been considered and approach the problem of multiple pathway feedback on a parallel bundle of relay cells.

\section{Formulation}

For this initial effort we will assume an overly simplified model so that the central results that develop can be examined without the confusion of undue detail. Extension to more complex formulations can then follow from these results.

There are a number of good studies of synaptic physiology in the literature. For the purposes of this report we mention three papers that present a comprehensive review of this area (Rall et al., 1967; Stevens, 1968; Kuno, 1971). The conclusions one might draw from these reports can be briefly summarized as follows: The transmission between presynaptic and postsynaptic neurons is quantal in nature, but in general the postsynaptic effects of the quanta sum linearly to produce the changes in membrane potential and, indeed, the effects of many presynaptic inputs to a neuron sum linearly to produce a change in membrane potential that is close to a linear weighted sum of the inputs. It seems that, in spite of the exceedingly complex machinery of synaptic transmission, we can as a first approximation represent a neuron as a slightly noisy linear summer of multiple inputs. We should add some linear membrane dynamics as well, perhaps of the form $t e^{-\alpha t}$ as suggested by Rall et al. (1967). 
We next assume that the "output" of each neuron is a weighted sum of the inputs including the various feedbacks that impinge on the particular neuron of note.

If it is necessary to invoke Dale's Law and insist that a given neuron can have only inhibitory or excitatory effects exclusively we will consider all neuron outputs to be excitatory and to insert an inverting interneuron to produce inhibitory feedback. The additional synaptic delay added by the interneuron will be lumped into the total latency of the compound fiber.

We will assume that each neuron in the pool produces a response that is the low pass filtered and reencoded version of the weighted sum of its "inputs". In doing this we ignore the fine structure of the spiketo-spike firing pattern of the cell and assume that the signal of interest, $y(t)$, is carried by a modulated point process. It has been shown by Knox (1970) and McKean et al. (1970) that the signal can be recovered without distortion by low pass filtering the modulated point process which has a Poisson counting function and is modulated by periodic signals. Yin (1973) has extended this to more generalized modulation conditions.

A given cell response results from the summation of a number of input spike trains. This tends to produce an output process that converges to a point process with a Poisson counting function almost regardless of the statistics of the constituent input spike trains. This has been confirmed specifically by Yin (1973) in his studies of thalamic responses to joint motion.

We propose, therefore, at least in certain specific cases, that the output of a given cell can be considered to be a signal term accompanied by a noise term, inherent in the nature of the point process that is carrying the signal. The noise term can be considered to be injected into the cell output and summed with the signal. The noises associated with each cell output are assumed to be independent. We will assume that the effect of the noise terms on the behavior of the system can be ignored at present by the virtue of two assumptions: 1. Since the summated noise terms add incoherently and the signal terms tend to add coherently the signal-to-noise ratio increases on the average by $\sqrt{n}$, where $n$ is the number of inputs to the cell; 2 . The assumptions of linearity allow us to assume that the total response at any point is the sum of the noise and signal contributions (superposition).

To describe this situation more succinctly consider Fig. 1. The variables $x_{1}, \ldots, x_{n}$ represent a set of independent external stimuli acting on the cell pool. The block labeled $H$ represents the mixing of external signals with signals generated by the cell's response to stimuli. The block labeled $D$ represents the various delay times of individual cell fibers. The block labeled

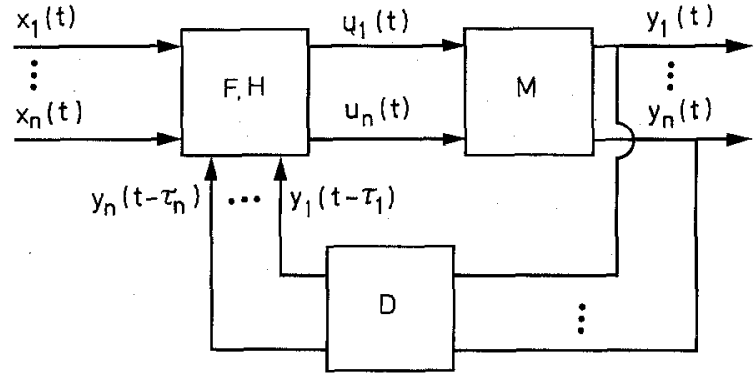

Fig. 1. A neural population with feedback

$M$ represents the response characteristic of the cell membrane.

The conceptual model of Fig. 1 must be sharpened somewhat before the analysis can proceed. In our study we shall invoke the following assumptions.

First the signal mixing block is linear. More explicitly we assume that there exist matrices with typical elements $h_{i j}(t)$ and $f_{i j}(t)$ such that

$u_{i}(t)=\sum_{j=1}^{n}\left(f_{i j}(t) x_{j}(t)-h_{i j}(t) y_{j}\left(t-\tau_{j}\right)\right)$.

Using vector notation: $x(t)=\left(x_{1}(t), \ldots, x_{n}(t)\right), y(t-\tau)=$ $\left(y_{1}\left(t-\tau_{1}\right), \ldots, y_{n}\left(t-\tau_{n}\right)\right)$ and $u(t)=\left(u_{1}(t), \ldots, u_{n}(t)\right)$. Equation (1) can be written in vector matrix form

$u(t)=F(t) x(t)-H(t) y(t-\tau)$.

Secondly we assume that $D$ is a multivariate linear array of delay lines, that is

$y(t-\tau)=(D y)(t)=\left(y_{1}(t-\tau), \ldots, y_{n}\left(t-\tau_{n}\right)\right)$.

Finally the behavior of the cell membrane, modeled by $M$, is taken to be linear. One acceptable form for $M$ is that of a time varying filter namely

$y(t)=(M u)(t)=\int_{0}^{t} \hat{M}(t, \beta) u(\beta) d \beta$

where $\hat{M}$ is a matrix valued impulse response function.

In the following section we take up the analysis of the system described by Eqs. (2), (3), and (4). Attention is focused on techniques for determining existence, uniqueness, and stability of solutions to these simultaneous equations and computational methods for iterating to the solution. In so doing some recent system theoretic results are brought to bear.

\section{Analysis}

Using the vector-matrix notation of Eqs. (2), (3), and (4) we have

$y=M(F x-H D y)$

or invoking the linearity of $M$ and $H$ we have

$y+M H D y=M F x$. 
For example with $M, H$, and $D$ computed as before, Eq. (5) is a family of $n$ equations whose $j^{\text {th }}$ member is of the form

$$
\begin{aligned}
& y_{j}(t)+\sum_{k, l=1}^{n} \int_{0}^{t} \hat{M}_{j k}(t, \beta) h_{k l}(\beta) y_{l}\left(\beta-\tau_{l}\right) d \beta \\
& \quad=\sum_{k, l=1}^{n} \int_{0}^{t} \hat{M}_{j k}(t, \beta) f_{k l}(\beta) x_{l}(\beta) d \beta \quad t \geqq 0
\end{aligned}
$$

Notice that in this model we tacitly assume $y(0)=0$.

Equation (5) points out that analysis of the neuron pool is intimately related to inversion of the operator $I+M H D$. Indeed if this operator is invertable then

$y=(I+M H D)^{-1} \cdot M F x$

is an explicit representation of the cell pool behavior.

Our cell pool analysis then leads naturally to the question as to properties of $M H D$ such that a solution to Eq. (5) exists, is unique, is stable, and can be computed by specified algorithms. The analysis of such questions has a rich history in the technical literature. We cannot hope to do justice to the many authors who have labored in this vineyard, however, there are a number of significant introductions to the underlying analytic machinery available at this writing (Brockett and Willems, 1965; Damborg and Naylor, 1970; DeSantis and Porter, 1974; DeSantis, 1971; Minty, 1964; Porter, 1966, 1971; Sandberg, 1964; Willems, 1971; and Zames, 1966).

Our attention is focused on using the recent results embodied in studies by DeSantis and Porter (1974) and DeSantis (1971). These studies utilize the structure of the abstract Hilbert resolution space. We shall interpret the abstract result in the present setting omitting the details of this transition for both brevity and readability.

The key result in its abstract form is summarized in the following lemma (DeSantis and Porter, 1974).

\section{Lemma 1}

If $T$ is a bounded linear strictly causal operator on a Hilbert resolution space then the $I-T$ is invertable and the inverse is computed by the uniformly convergent series

$(I-T)^{-1}=\sum_{j=0}^{\infty} T^{j}$.

Here $T^{0}=I, T^{1}=T, T^{2}=T \cdot T$, and $T^{n}=T \cdot T^{n-1}$.

Returning now to the operators of Eqs. (2), (3), and

(4) consider the finite interval $[0, k]=\{0 \leqq t \leqq k\}$. Then the following assumptions and conclusions can be brought to bear.

\section{Assumption 1}

Suppose each $h_{i j}$ of Eq. (1) is bounded and sectionally continuous on $[0, k]$. Then the map $H$ is bounded and causal.

\section{Assumption 2}

Suppose that $\tau_{j}>0 j=1, \ldots, n$ in Eq. (3). Then $D$ is bounded and strictly causal.

\section{Assumption 3}

Suppose $\hat{M}_{j k}(t, \beta)=0$ if $\beta>t$ and that $\int_{0}^{k} \int M_{j k}^{2}(t, \beta) d t d \beta<\infty$ all $j, k$ then $M$ is bounded and causal.

\section{Proposition 1}

If $H, D$, and $M$ satisfy Assumptions 1, 2, and 3 respectively then $M H D$ is bounded linear and strictly causal.

\section{Proof}

The proof of this proposition follows easily from the fact that the composition of bounded linear functions is a bounded linear function and the fact that the composition of a causal function with a strictly causal linear function is strictly causal (DeSantis and Porter, 1974).

A direct consequence of Lemma 1 and Proposition 1 is the following.

\section{Corollary}

If $H, D$, and $M$ satisfy Assumptions 1,2, and 3 respectively then for every square integrable stimulus, $x$, there exists a unique square integrable response, $y$, determined by Eq. (7). Moreover

$(I+M H D)^{-1}=\sum_{j=0}^{\infty}(-M H D)^{j}$,

where the convergence is uniform.

Our corollary can be given an intuitive interpretation. If $\min \left\{\tau_{j}\right\}=\tau>0$ then if $\tau n \geqq k$ it is easily seen that $D^{n}$ is the zero operator on the interval $[0, k]$. Similarly $(M H D)^{n}=0$ on $[0, k]$ and hence the series of the corollary terminates finitely and hence converges. It remains only to show that it converges to $(I+M H D)^{-1}$. To see this note that by operating with $(I+M H D)$ on both sides of Eq. (8) we have

$$
\begin{aligned}
& (I+M H D)\left[I-M H D+(M H D)^{2}-(M H D)^{3}+\ldots\right] \\
& \quad=I-M H D+M H D+(M H D)^{2}-(M H D)^{2}+\ldots=I .
\end{aligned}
$$

On infinite intervals $[0, \infty]$ the result is still true. First the series does terminate finitely on all finite subintervals. Moreover the square integrability of Assumption 3 assists in producing convergence in the limit as $k \rightarrow \infty$.

\section{Conclusions}

We have shown that solutions to the formulation embodied by Eq. (7) exists, is unique, is square integrable and can be computed by the specified algorithms. 


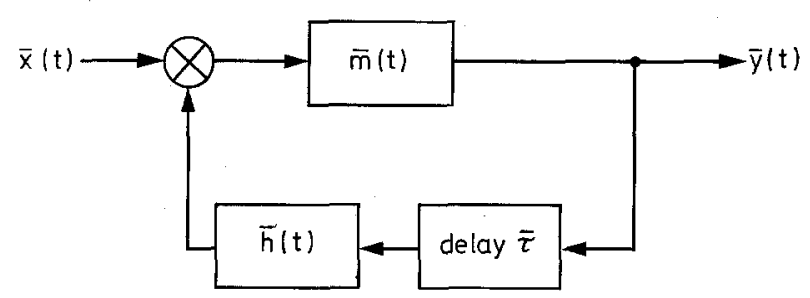

Fig. 2. Simplified system

Further implications can be drawn from the results as well. Some of these implications are not intuitively obvious when simpler systems formulations are utilized. Consider the system of Fig. 2, for example. This is the result of an attempt to "lump" the salient features of the system into a few black boxes.

Bending our system formulation to this model would produce an average cell membrane characteristic $\bar{h}$ an average feedback delay $\bar{\tau}$ and a single summing junction as shown in Fig. 2.

This simplified type of formulation can be explicitly or implicitly found in many studies of neurological systems and can produce useful insights into the behavior of the system under study. Stark (1968) has reported several pioneering efforts along these lines.

Systems with feedback can become unstable and produce oscillations. Oscillations in the neuromuscular system (clonus) and the pupillary control system (hippus) can occur under pathological conditions. Oscillations in other neurological systems (e.g., respiratory control) can produce the natural rhythm required for system function, according to Cohen (1973).

The model of Fig. 2 suggests that oscillation would occur if the gain and phase shift in the loop were properly adjusted. Determinations of such conditions of oscillation are well known in classical control theory. Oscillation would always be predicted for a sufficiently high loop gain in such a system.

Can the system of Fig. 1 with Assumptions 1, 2, and 3 produce an oscillatory behavior? The answer to this question is yes. On a finite time interval, $[0, k]$, the system response can be oscillatory and this oscillatory behavior will be computable by the series of Eq. (8). Naturally, the mode of oscillation can be much more complex than the modes available in the simplified system.

It is perhaps worthwhile to point out the fact that when one is observing a given $y(t)$ as in single unit neurophysiology one is observing the manifestation of the properties of virtually all of the neurons in the pool and all inputs to the neuron pool. This can readily be seen in Eq. (7). Any element of the $y$ vector results from products and summations of the properties of all cells and all inputs to the neuron pool. This should be obvious without the model development, but Eq. (7) helps focus our attention on this fact.

Our formulation at present is valid for situations where reasonably linear conditions apply. The nervous system, once the nonlinearities of the peripheral end organs have been broached, is surprisingly linear in many cases. Mountcastle and his co-workers (Mountcastle, Talbot and Kornhuber, 1966) have observed similarities in the response characteristics of cutaneous receptors and human subjects when equivalent stimulus paradigms were applied to the skin. This finding has led them to the conclusion that the intervening transformations between receptor response and subjective sensory events at higher levels may be linear in nature. Terzuolo and his associates have shown that the neuromuscular system is rather linear in its system behavior even though significant nonlinearities are observed in the responses of muscle and receptor units (Poppele and Terzuolo, 1968; Rosenthal et al., 1970; Roberts et al., 1971). Even when linear conditions do not hold, their basic approach yields useful insights into understanding human performance in motor tasks (Soechting, 1973; Viviano and Terzuolo, 1973).

Our own theoretical development is motivated by ongoing experimental studies of the joint receptor system (Williams et al., 1973). There are significant second order nonlinearities in a class of rapidly adapting receptors in this system. It seems that the Volterra formalism (Poggio and Reichardt, 1973; Rice, 1973) can extend the formulation presented in this paper. The work of George (1959) and Zames (1960) is also of interest because of the use of the delay operator in its iteration procedure.

Newly developed experimental techniques may allow one to observe multiple inputs to a nucleus of cells (Heetderks and Williams, 1975). Thus the elements of the input vector $x$ and at least one element of the $y$ vector may soon become available in an experimental setting. The formulation developed in the present paper or an extended version would then be of use in the analysis of such data.

\section{References}

Brockett, R., Willems, J.: Frequency domain stability criteria. Part I, IEEE Trans. on Automatic Control, AC-10, 3, 255-261 (1965); Part II, AC-10, 4, 407-413 (1965)

Carpenter, D., Henneman, E.: Size threshold relation in receptors. J. Neurophysiol. 9, 353-368 (1966)

Coggshall, J.: Linear models for biological transducers and impulse train spectra: General information and review. Kybernetik 13, $30-37(1973)$ 
Coggshall, J., Bekey, G.: A stochastic model of skeletal muscle based on motor unit properties. Math. Biosci. 7, 405-419 (1970)

Cohen, M.: Neurons and neuron populations: Commentary, Regulation and control in physiological systems, pp. 520-521. A.Iberall and A.Guyton (Eds.), Pittsburgh, Pa.: ISA Press 1973

Cohen, M.: Dynamics of networks which generate respiratory rhythmicity. Regulation and control in physiological systems, pp. 530-532. A. Iberall, A.Guyton (Eds.), Pittsburgh, Pa.: ISA Press 1973

Damborg, M., Naylor, A.: The fundamental structure of input-output stability for feedback systems. IEEE Trans. Syst. Sci. Cybern. SSC-6, 92-96 (1970)

DeSantis, R., Porter,W.: On the generalization of the Volterra principal of inversion. J. Math. Analy. Appl. 3, 48 (1974)

DeSantis, R.: Causality and stability in resolution space. Proc. of the 14th Midwest Symposium on Circuit Theory, University of Denver, 115-121 (1971)

George,D.A.: Continuous nonlinear systems. Mass. Inst. of Technology, Research Laboratory for Electronics Report, 355 (1959)

Heetderks, W.J., Williams, W. J.: Partition of gross peripheral nerve activity into single unit responses by correlation techniques. Science 188, 373-375 (1975)

Henneman, E., Somjen, G., Carpenter, D.: Excitability and inhibitibility of motoneurons of different sizes. J. Neurophysiol. 28, $599-620(1965)$

Henneman, E., Somjen, G., Carpenter, D.: Functional significance of cell size in spinal motoncurons. J. Neurophysiol. 28, 560-580 (1965)

Johnson, K.: Reconstruction of population response to a vibratory stimulus in quickly adapting mechano-receptive afferent fiber population innervating glabrous skin of the monkey. J. Neurophysiol. 37, 48-72 (1974)

Knox,C.: Signal transmission in random spike trains with applications to the statocyst neurons of the lobster. Kybernetik 7, $167-174$ (1970)

Kuno, M.: Quantal synaptic transmission. Physiol. Rev. 51, 647—678 (1971)

McKean, T.A., Poppele, R.E., Rosenthal, N. P., Terzuolo, C.A.: The biologically relevant parameter in nerve impulse trains. Kybernetik 6, 168-170 (1970)

Minty, $G$.: On the solvability of nonlinear functional equations of monotonic type. Pacific J. Math. 6, 249-255 (1964)

Mountcastle,V.B., Talbot, W.H., Werner,G.: The neural transformation of mechanical stimuli delivered to the monkey's hand. In: de Reuck, A. S. V., Knight, J. (Eds.): Touch, heat and pain (Ciba Foundation Symposium). London: Churchill 1966

Poggio, T., Reichardt,W.: Considerations on models of movement detection. Kybernetik 13, 223-227 (1973)

Poppele,R.E., Terzuolo,C.A.: Myotatic reflex: Its input-output relation. Science 159, 743--745 (1968)

Porter,W.: Modern Foundations of Systems Engineering. New York: MacMillan \& Co. 1966

Porter, W.: Nonlinear systems in Hilbert space. Int. J. Contr. 13, $20-44(1971)$
Rall, W., Burke, R. E., Smith, T.G., Nelson, P.G., Frank, K.: Dentritic location of synapses and possible mechanisms for the monosynaptic EPSP in motoneurons. J. Neurophysiol. 30, 1169—1193 (1967)

Roberts, W.J., Rosenthal, N.P., Terzuolo, C.A.: A control model of stretch reflex. J. Neurophysiol. 34, 620-634 (1971)

Rosenthal, N. P., McKean, T.A., Roberts, W.J., Terzuolo, C.A.: Frequency analysis of stretch reflex and its main subsystems in triceps surae of the cat. J. Neurophysiol. 33, 713-749 (1970)

Sandberg, $\mathrm{I}_{\text {: }}$ : On the $\mathrm{L}_{2}$-boundedness of solutions of nonlinear functional equations. Bell Syst. Techn. J. 43, 1581-1599 (1964)

Soechting, J.F.: Modeling of a simple motor ask in man. Motor output dependence on sensory inputs. Kybernetik 14, 25-34 (1973)

Stark, L.: Neurological Control Systems. New York: Plenum Press 1968

Stevens, C.F.: Synaptic physiology. Proc. IEEE 56, 916930 (1968)

Towe,A.L.: Neuronal population behavior in the somatosensory systems. In: Kenshalo,D. (Ed.): The skin senses, pp. 552-574. Springfield, Ill.: C. C. Thomas 1968

Viviani, P., Terzuolo,C.A.: Modeling of a motor task in man. Kybernetik 35, 35-62 (1973)

Whitehorn, D., Bromberg, M., Howe,J., Putnam, P.: Activation of gracile nucleus: Time distribution of activity in presynaptic and postsynaptic elements. Exp. Neurol. 37, 312-321 (1972)

Willems, J.: Analysis of Feedback Systems. Cambridge: M.I.T. Press 1971

Williams, W.: Biocybernetic aspects of latency dispersion in peripheral nerve bundles in sensory and motor systems. Proc. IEEE Syst. Cybern. Conf. 6-12, 135-146 (1968)

Williams, W.: Transfer characteristics of two tandem nerve bundles. Proc. 8th Intern. Conf. Eng. Med. Biol. 27-7 (1969)

Williams, W.: Transfer characteristics of dispersive nerve bundles. IEEE Trans. Syst. Man Cybern., SMC-2, 72-85 (1972)

Williams, W., Heetderks, W.: Transfer characteristics of heterogeneous populations of neural elements. In: Guyton, A., Iberall,A. (Eds.): Regulation and control in physiological systems, pp. 527539. Pittsburgh, Pa.: ISA Press 1973

Williams, W., BeMent, S., Yin, T., McCall,W.,Jr.: Nucleus gracilis responses to joint motion: A frequency response study. Brain Res. 64, 123-140 (1973)

Yin, T.: Dynamic response and transfer characteristics of knee joint afferents in somatosensory thalamus of the cat. Ph. D. Dissertation, The University of Michigan, Ann Arbor, Mich. 1973

Zames, G.: Nonlinear operations for Systems analysis. Sc.D. Thesis, Mass. Inst. of Technology, Cambridge, Mass. 1960

Zames, G.: On the input-output stability of time-varying nonlinear feedback systems-Part II: Conditions involving circles in the frequency plane and sector nonlinearities. IEEE Trans. Autom. Contr. AC-11, 3, 465-476 (1966)

Prof. W. A. Porter

Dept. of Electrical and Computer Engineering

The University of Michigan Ann Arbor, Mich. 48104, USA 